\title{
Fuzzy Convergence of Fuzzy Complex Series
}

\author{
Pishtiwan O. Sabir ${ }^{1}$, Adil K. Jabbar ${ }^{2}$, Munir A. Al-Khafagi ${ }^{3}$ \\ ${ }^{1,2}$ Department of Mathematics, Faculty of Science and Education Sciences, University of \\ Sulaimani, Sulaimani, Kurdistan-Iraq \\ ${ }^{3}$ Department of Mathematics, College of Education, University of Al-Mustansiriya, Bagdad, Iraq \\ ${ }^{1}$ pishtiwan.sabir@univsul.edu.iq, ${ }^{2}$ adilkj@gmail.com, ${ }^{3}$ mnraziz@yahoo.com
}

\begin{abstract}
In this paper, based on $\left[\gamma^{\star}, \gamma^{\star \star}\right]$-level converges some important results on fuzzy series of generalized rectangular valued bounded closed complex complement normalized fuzzy numbers are proved.
\end{abstract}

Keywords: Fuzzy Complex Numbers; Fuzzy Sequences; Fuzzy series

\section{Introduction, Definitions and Notations}

It is known that the concept of fuzzy complex analysis was first considered by Buckley and Qu [1] which is used the definition of the derivative of a fuzzy function, which maps the open interval $(a, b)$ into the fuzzy subset of the real $F(R)$ in [5], to generalize a fuzzy function maps $(a, b)$ into the set of fuzzy subsets of the complex case $F(\mathbb{C})$. Buckley in [2] defined a metric on the set of complex fuzzy numbers to provide the necessary background and allowed us to discuss continuities, convergences, and differentiation of complex fuzzy function $[3,8,13$ $18,24]$. In view of [2], Guangquan [7] discussed the limit theory of the sequence of fuzzy complex numbers in detail, giving some results about limit theory, which are the counterparts of well-known results valid for real numbers in classical mathematics analysis. Buckley's work consummate continued research and scholars did many works about the set of complex fuzzy sets that extensively studied fuzzy analysis such as $[6,9,11,19,23]$.

Zadeh [20] defined fuzzy set $\tilde{A}$ on the universal set $X$, which is a mapping $\mu_{\tilde{A}}(x): X \rightarrow[0,1]$. He defined the strong $\alpha$-cut ${ }^{\alpha+} \tilde{A}$, the weak $\alpha-$ cut, ${ }^{\alpha-} \tilde{A}$, the height, $\alpha_{\tilde{A}}^{\max }$, and the core, $\operatorname{core}(\tilde{A})$ of the fuzzy set $\tilde{A}$, respectively, as the non- 
fuzzy set of all elements of the universal set $X$ that belongs to the fuzzy set $\tilde{A}$ at least to the degree $\alpha \in[0,1]$; the ordinary set that contains all elements of the universal set whose membership grades in the given set are greater than but do not include the specified value of $\alpha$; the largest value of $\alpha$ for which the $\alpha$-cut is not empty; the non-fuzzy set of all points $x$ in the universal set $X$ at which $\sup _{x} \mu_{\tilde{A}}(x)$ is essentially attained. Frequently, we will write $\mu(x, \tilde{A})$ instead of $\mu_{\tilde{A}}(x)$ and shall use the fuzzy power set on $\mathrm{X}$ by $\mathcal{F}(X)$. Let $\tilde{A} \in \mathcal{F}(X)$. If $r$ is any nonnegative real number such that $\left(r \sup _{x \in X} \mu(x, \tilde{A})\right) \leq 1$, then $\mu(x, r \tilde{A})=r \mu(x, \tilde{A})$, for all $x \in X$.

Let $\tilde{A}_{i} \in \mathcal{F}(X), i \in I$ ( $I$ is a nonempty index set). Then the standard fuzzy union of $\tilde{A}_{i}, \mathrm{U}_{i} \tilde{A}_{i}$, is defined by $\sup _{x} \mu\left(x, \tilde{A}_{i}\right)=\mathrm{V}_{x} \mu_{\tilde{A}_{i}}(x)$, the standard fuzzy intersection of $\tilde{A}_{i}, \bigcap_{i} \tilde{A}_{i}$, is defined by $i n f_{x} \mu\left(x, \tilde{A}_{i}\right)=\Lambda_{x} \mu_{\tilde{A}_{i}}(x)$, and the complement of $\tilde{A}_{i}, \neg \tilde{A}_{i}$, is defined by $\mu\left(x, \neg \tilde{A}_{i}\right)=1-\mu\left(x, \tilde{A}_{i}\right)$, for all $x \in X$.

Extension principle for fuzzy sets was first presented by Zadeh [21, 22], which provides a method for extending classical mathematical concepts to fuzzy quantities. Let $f: X_{1} \times X_{2} \times \ldots \times X_{n} \rightarrow Y$ be given by $y=f\left(x_{1}, x_{2}, \ldots, x_{n}\right)$ and $\tilde{A}_{i} \in \mathcal{F}\left(X_{i}\right)$ for $i=1,2, \ldots n$. Here the fuzzy set $\tilde{C}=f\left(\tilde{A}_{1}, \tilde{A}_{2}, \ldots, \tilde{A}_{n}\right)$ is defined by

$$
\tilde{C}(y)=\bigvee_{x_{1}, x_{2}, \ldots, x_{n} \mid y=f\left(x_{1}, x_{2}, \ldots, x_{n}\right)}\left(\mu\left(x_{1}, \tilde{A}_{1}\right) \wedge \mu\left(x_{2}, \tilde{A}_{2}\right) \wedge \ldots \wedge \mu\left(x_{n}, \tilde{A}_{n}\right)\right)
$$

A fuzzy number was first introduced by Chang and Zadeh [4] characterized by a grade of membership $\mu(x, \tilde{a}): \mathbb{R} \rightarrow[0,1]$ satisfies:

1. $\mu(x, \tilde{a}): \mathbb{R} \rightarrow[0,1]$ is upper semi-continuous (u.s.c);

2. There are $a \in \mathbb{R}$ and $b \in \mathbb{R}$ such that $c \leq a \leq b \leq d$;

3. $\mu(x, \tilde{a})$ is increasing on the interval $[\mathrm{c}, \mathrm{a}]$;

4. $\mu(x, \tilde{a})$ is decreasing on the closed interval $[b, d]$;

5. $\mu(x, \tilde{a})=0$ outside some interval $[c, d]$;

6. $\mu(x, \tilde{a})=1$ for the interval $[a, b]$.

We will use $\mathcal{F}^{\star}$ to denote the set of all fuzzy numbers on $\mathbb{R}$. 
Qiu et al. [12] redefine a fuzzy complex number $\tilde{Z}$ by its membership function $\mu(z, \tilde{Z}): \mathbb{C} \rightarrow[0,1]$ which satisfies:

1. $\mu(z, \tilde{Z})$ is continuous;

2. ${ }^{\alpha-} \tilde{Z}, 0 \leq \alpha<1$, is open, bounded, and connected;

3. $\{z \mid \mu(z, \tilde{Z})=1\}$ is non-empty, compact, and arcwise connected. We use $\mathcal{F}^{\star \star}$ for the power set of fuzzy complex numbers.

Qiu et al. [12] generalized a complex fuzzy number $\tilde{Z}$ if and only if:

1. $\mu(z, \tilde{Z}): \mathbb{C} \rightarrow[0,1]$ is u.s.c.;

2. ${ }^{\alpha+} \tilde{Z}, 0 \leq \alpha \leq 1$, is compact and arcwise connected;

3. ${ }^{1+} \tilde{Z}$ is non-empty.

Let $\tilde{Z}^{\prime}, \tilde{Z}^{\prime \prime} \in \mathcal{F}^{\star \star}$. If we denote the extended operation by $\circledast$, then by the extension principle, one obtains

$$
\begin{aligned}
& \mu\left(z, \tilde{Z}^{\prime} \oplus \tilde{Z}^{\prime \prime}\right)=\sup _{z=z^{\prime}+z^{\prime \prime}} \min \left(\mu\left(z^{\prime}, \tilde{Z}^{\prime}\right), \mu\left(z^{\prime \prime}, \tilde{Z}^{\prime \prime}\right)\right), \text { for all } z \in \mathbb{C} \\
& \mu\left(z, \tilde{Z}^{\prime} \odot \tilde{Z}^{\prime \prime}\right)=\sup _{z=z^{\prime} \cdot z^{\prime \prime}} \min \left(\mu\left(z^{\prime}, \tilde{Z}^{\prime}\right), \mu\left(z^{\prime \prime}, \tilde{Z}^{\prime \prime}\right)\right), \text { for all } z \in \mathbb{C}
\end{aligned}
$$

For complement normalized fuzzy numbers (CNFNs) [10] $\tilde{\mu}$ and $\tilde{\lambda}$ with membership functions $\mu\left(\tilde{\mu}, \mu_{1}\right)$ and $\mu\left(\tilde{\lambda}, \lambda_{1}\right)$, respectively, we call $\tilde{Z}=\tilde{\mu} \boxplus i \tilde{\lambda}$ a bounded closed complex complement normalized fuzzy number (BCCCNFN) with membership function $\mu(\tilde{Z}, z)=\mu\left(\tilde{\mu}, \mu_{1}\right) \vee \mu\left(\tilde{\lambda}, \lambda_{1}\right)$, where $z=\mu_{1}+i \lambda_{1}$. We denote the class of all the BCCCNFNs by $\tilde{\mathcal{F}}_{\neg N}^{* *}$ and use $\tilde{\mathcal{F}}_{\neg N}^{*}$ for the fuzzy power set of CNFNs.

We call $\mu_{[\widetilde{\mu}]}(\delta): \mathbb{R} \rightarrow\left\{\left[\gamma^{-}, \gamma^{+}\right] ; \gamma^{-} \leq \gamma^{+}\right.$and $\left.\gamma^{-}, \gamma^{+} \in\left[0, \alpha_{\widetilde{\mu}}^{\max }\right]\right\}$ is a generalized CNFNs if $\mu_{[\widetilde{\mu}]}(\delta)=\left[\mu_{\widetilde{\mu}^{l}}(\delta), \mu_{\widetilde{\mu}^{u}}(\delta)\right]$ for $\tilde{\mu}^{l}, \tilde{\mu}^{u} \in \tilde{\mathcal{F}}_{\neg N}^{*}$. We call $\lambda([\tilde{Z}], \delta+$ $i \Delta): \mathbb{C} \rightarrow\left\{\left[\gamma^{-}, \gamma^{+}\right]:\left(\gamma^{-}, \gamma^{+}\right) \in\left[0, \alpha_{\tilde{Z}}^{\max }\right]^{2} ; \gamma^{-} \leq \gamma^{+}\right\}$is a generalized rectangular valued bounded closed complex complement normalized fuzzy number $(\mathrm{GRVBCCCNFN}) \quad$ if $\quad \lambda([\tilde{Z}], \delta+i \Delta)=[\lambda(\underline{\tilde{Z}}, \delta+i \Delta), \lambda(\overline{\tilde{Z}}, \delta+i \Delta)] \quad$ for BCCCNFNs $\underline{\tilde{Z}}$ and $\overline{\tilde{Z}}$. Sometime, we write $[\tilde{Z}]$ to be $[\underline{\tilde{Z}}, \overline{\tilde{Z}}]=[\tilde{\mu}][+] i[\tilde{\lambda}]=$ $\left[\tilde{\mu}^{\ell} \boxplus i \tilde{\lambda}^{\ell}, \tilde{\mu}^{u} \boxplus i \tilde{\lambda}^{u}\right]$, and we say $[\tilde{Z}]=[\widetilde{W}]=[\tilde{\gamma}][+] i[\tilde{\beta}]$ if ${ }^{\left[\gamma^{\star}, \gamma^{\star \star}\right]+}[\tilde{\mu}]=$ $\left[\gamma^{\star}, \gamma^{\star \star}\right]+[\tilde{\gamma}]$ and ${ }^{\left[\gamma^{\star}, \gamma^{\star \star}\right]+}[\tilde{\lambda}]={ }^{\left[\gamma^{\star}, \gamma^{\star \star}\right]+}[\tilde{\beta}]$. The set of all GRVBCCCNFNs is denoted by $\left[\tilde{\mathcal{F}}_{\neg N}^{* *}\right]$. We define a function $\tilde{\Gamma}$ on GRVBCCCNFNs $[\underline{\tilde{z}}, \overline{\tilde{Z}}]$, and $[\underline{\widetilde{W}}, \overline{\widetilde{W}}]$ 
as $\quad \tilde{\Gamma}([\underline{\tilde{Z}}, \overline{\tilde{Z}}],[\underline{\widetilde{W}}, \overline{\widetilde{W}}])=\left[\tilde{\xi}\left(\tilde{\mu}^{\ell}, \tilde{\gamma}^{\ell}\right) \vee \tilde{\xi}\left(\tilde{\lambda}^{\ell}, \tilde{\beta}^{\ell}\right), \tilde{\xi}\left(\tilde{\mu}^{u}, \tilde{\gamma}^{u}\right) \vee \tilde{\xi}\left(\tilde{\lambda}^{u}, \tilde{\beta}^{u}\right)\right]$, where $\tilde{\xi}\left(\tilde{\mu}^{\ell}, \tilde{\gamma}^{\ell}\right)=\int_{0}^{\alpha_{\widetilde{\gamma}^{\ell}, \tilde{\mu}^{\ell}}^{\max }} \gamma\left[\left|\alpha_{\widetilde{\gamma}^{\ell}, \tilde{\mu}^{\ell}}^{\max } \tilde{\mu}^{\ell^{-}}-{ }^{\alpha_{\widetilde{\gamma}^{l}, \tilde{\mu}^{\ell}}^{\max }} \tilde{\gamma}^{\ell^{-}}\right|,\left.\vee_{\gamma \leq \psi \leq \alpha_{\widetilde{\gamma}^{\ell},,^{\ell}}}\right|^{\psi+} \tilde{\mu}^{\ell^{-}}-{ }^{\psi+} \tilde{\gamma}^{\ell^{-}} \mid \vee\right.$ $\left.\left|{ }^{\psi+} \tilde{\mu}^{\ell^{+}}-{ }^{\psi+} \tilde{\gamma}^{\ell^{+}}\right|\right]$. Let $[\tilde{Z}] \in\left[\tilde{\mathcal{F}}_{\neg N}^{* *}\right]$ and $\left(\gamma^{-}, \gamma^{+}\right) \in I_{[0}^{1[} \times I_{[0}^{1[}$. We define $\left[\gamma^{-}, \gamma^{+}\right]-$ level of $[\tilde{Z}]$ as ${ }^{\left[\gamma^{-}, \gamma^{+}\right]+}[\tilde{Z}]=\gamma^{\gamma^{+}} \underline{\tilde{Z}} \cap \gamma^{\gamma^{+}+} \overline{\tilde{Z}}$.

\section{Fuzzy Convergence of GRVBCCCNFNs}

Throughout this section, some important results on series of GRVBCCCNFNs based on $\tilde{\Gamma}$-converge and $\left[\gamma^{\star}, \gamma^{\star \star}\right]$-level converges are examined and proved.

Definition 2.1. Let $\left(\left[\tilde{Z}_{\eta}\right]=\left[\tilde{\mu}_{\eta}\right][+] i\left[\tilde{\lambda}_{\eta}\right]\right)$ be a sequence in $\left[\tilde{\mathcal{F}}_{\neg N}^{* *}\right],[\widetilde{W}]=$ $[\tilde{\gamma}][+] i[\tilde{\beta}] \in\left[\tilde{\mathcal{F}}_{\neg N}^{* *}\right]$. We say that $\left(\left[\tilde{Z}_{\eta}\right]\right)$ is $\tilde{\Gamma}$-converges to $[\widetilde{W}]$ written as $\left(\left[\tilde{Z}_{\eta}\right]\right) \stackrel{\widetilde{\Gamma}}{\longrightarrow}[\widetilde{W}]$, if for any $\bar{\varepsilon}=[\varepsilon, \varepsilon]>\overline{0}$, there exists a positive integer $N$ such that $\tilde{\Gamma}\left(\left[\tilde{Z}_{\eta}\right],[\widetilde{W}]\right)<\bar{\varepsilon}$ as $\eta \geq N$.

Definition 2.2. Let $\left(\left[\tilde{Z}_{\eta}\right]=\left[\tilde{\mu}_{\eta}\right][+] i\left[\tilde{\lambda}_{\eta}\right]\right) \subset\left[\tilde{\mathcal{F}}_{\neg N}^{* *}\right],[\tilde{Z}]=[\tilde{\gamma}][+] i[\tilde{\beta}] \in\left[\tilde{\mathcal{F}}_{\neg N}^{* *}\right]$ and $\left(\gamma^{\star}, \gamma^{\star \star}\right) \in I_{[0}^{1[} \times I_{[0}^{1[}$. We say that $\left(\left[\tilde{Z}_{\eta}\right]\right)$ is $\left[\gamma^{\star}, \gamma^{\star \star}\right]$-level converges to $[\tilde{Z}]$ written as $\left(\left[\tilde{Z}_{\eta}\right]\right) \stackrel{\left[\gamma^{\star}, \gamma^{\star \star}\right]-\text { level }}{\longrightarrow}[\tilde{Z}]$, if ${ }^{\gamma^{\star}+} \tilde{\mu}_{\eta}^{l-} \mathrm{\vee}^{\gamma^{\star \star}+} \tilde{\mu}_{\eta}^{u-} \longrightarrow \gamma^{\star}+\tilde{\gamma}^{l^{-}} \mathrm{\vee}^{\gamma^{\star \star}+} \tilde{\gamma}^{u^{-}}$, $\gamma^{\star}+\tilde{\mu}_{\eta}^{l+} \wedge^{\gamma^{\star \star}+} \tilde{\mu}_{\eta}^{u+} \longrightarrow{ }^{\gamma^{\star}+} \tilde{\gamma}^{l^{+}} \wedge^{\gamma^{\star \star}+} \tilde{\gamma}^{u^{+}}, \quad \gamma^{\star}+\tilde{\lambda}_{\eta}^{l-} \vee^{\gamma^{\star \star}+} \tilde{\lambda}_{\eta}^{u^{-}} \longrightarrow{ }^{\gamma^{\star}+} \tilde{\beta}^{l^{-}} \vee$ $\gamma^{\star \star}+\tilde{\beta}^{u^{-}}$and ${ }^{\gamma^{\star}+} \tilde{\lambda}_{\eta}^{l+} \wedge^{\gamma^{\star \star}+} \tilde{\lambda}_{\eta}^{u^{+}} \longrightarrow{ }^{\gamma^{\star}+} \tilde{\beta}^{l^{+}} \wedge^{\gamma^{\star \star}+} \tilde{\beta}^{u^{+}}$.

Definition 2.3. Let $\left(\left[\tilde{Z}_{\eta}\right]=\left[\tilde{\mu}_{\eta}\right][+] i\left[\tilde{\gamma}_{\eta}\right]\right) \subset\left[\tilde{\mathcal{F}}_{\neg N}^{* *}\right] \quad, \quad\left[\tilde{\beta}_{\eta}\right]=\widetilde{\sum_{n=1}^{\eta}}\left[\tilde{Z}_{n}\right]=$ $\widetilde{\sum_{n=1}^{\eta}}\left[\tilde{\mu}_{n}\right][+] i \widetilde{\sum_{n=1}^{\eta}}\left[\tilde{\gamma}_{n}\right]$. If there exists $[\tilde{Z}]=[\tilde{\mu}][+] i[\tilde{\gamma}] \in\left[\tilde{\mathcal{F}}_{\neg N}^{* *}\right]$ such that $\left(\left[\tilde{\beta}_{\eta}\right]\right) \stackrel{\left[\gamma^{\star}, \gamma^{\star \star}\right]-\text { level }}{\longrightarrow}[\tilde{Z}]$, we say the GRVBCCCNFNs series (GRVBCCCNFNS) $\widetilde{\sum_{\eta=1}^{\infty}}\left[\tilde{Z}_{\eta}\right]$ is fuzzy converge to $[\tilde{Z}]$, and denoted by $\widetilde{\sum_{\eta=1}^{\infty}}\left[\tilde{Z}_{\eta}\right]=[\tilde{Z}]$.

Theorem 2.4. Let $\left(\left[\tilde{Z}_{\eta}\right]\right) \subset\left[\tilde{\mathcal{F}}_{\neg N}^{* *}\right],[\widetilde{W}] \in\left[\tilde{\mathcal{F}}_{\neg N}^{* *}\right]$. Then $\left(\left[\tilde{Z}_{\eta}\right]\right) \stackrel{\widetilde{\Gamma}}{\longrightarrow}[\widetilde{W}]$ if and only if $\left(\left[\widetilde{Z}_{\eta}\right]\right)$ is $\left[\gamma^{\star}, \gamma^{\star}\right]$-level converges to $[\widetilde{W}]$. 
Proof: For any $\bar{\varepsilon}>\overline{0}$, there exists a positive integer $N$ such that $\tilde{\Gamma}\left(\left[\tilde{Z}_{\eta}\right],[\widetilde{W}]\right) \prec \bar{\varepsilon}$ as $\eta \geq N$. This implies that, $\tilde{\xi}\left(\tilde{\mu}_{\eta}{ }^{\ell}, \tilde{\gamma}^{\ell}\right) \prec \varepsilon, \tilde{\xi}\left(\tilde{\lambda}_{\eta}{ }^{\ell}, \tilde{\beta}^{\ell}\right) \prec \varepsilon, \tilde{\xi}\left(\tilde{\mu}_{\eta}{ }^{u}, \tilde{\gamma}^{u}\right) \prec \varepsilon$, and $\tilde{\xi}\left(\tilde{\lambda}_{\eta}{ }^{u}, \tilde{\beta}^{u}\right) \prec \varepsilon$ if and only if $\left|{ }^{\psi+} \tilde{\mu}_{\eta}{ }^{\ell^{-}}-{ }^{\psi+} \tilde{\gamma}^{\ell^{-}}\right| \vee\left|{ }^{\psi+} \tilde{\mu}_{\eta}{ }^{\ell^{+}}-{ }^{\psi+} \tilde{\gamma}^{\ell^{+}}\right|<\varepsilon$, $\left|{ }^{\psi+} \tilde{\mu}_{\eta}{ }^{\ell^{-}}-{ }^{\psi+} \tilde{\beta}^{\ell^{-}}\right| \mathrm{\vee}\left|{ }^{\psi+} \tilde{\mu}_{\eta}{ }^{\ell^{+}}-{ }^{\psi+} \tilde{\beta}^{\ell^{+}}\right|<\varepsilon, \quad\left|{ }^{\psi+} \tilde{\mu}_{\eta}{ }^{u^{-}}-{ }^{\psi+} \tilde{\gamma}^{u-}\right| \vee \mid{ }^{\psi+} \tilde{\mu}_{\eta}{ }^{u^{+}}-$ ${ }^{\psi+} \tilde{\gamma}^{u+} \mid<\varepsilon$, and $\left|{ }^{\psi+} \tilde{\mu}_{\eta}{ }^{u-}-{ }^{\psi+} \tilde{\beta}^{u^{-}}\right| \vee\left|{ }^{\psi+} \tilde{\mu}_{\eta}{ }^{u^{+}}-{ }^{\psi+} \tilde{\beta}^{u^{+}}\right|<\varepsilon$ if and only if ${ }^{\psi+} \tilde{\mu}_{\eta}{ }^{\ell^{-}} \longrightarrow{ }^{\psi+} \tilde{\gamma}^{\ell^{-}} \quad, \quad{ }^{\psi+} \tilde{\mu}_{\eta}{ }^{\ell^{+}} \longrightarrow{ }^{\psi+} \tilde{\gamma}^{\ell^{+}}, \quad{ }^{\psi+} \tilde{\mu}_{\eta}{ }^{\ell^{-}} \longrightarrow{ }^{\psi+} \tilde{\beta}^{\ell^{-}}$, ${ }^{\psi+} \tilde{\mu}_{\eta}{ }^{\ell^{+}} \longrightarrow{ }^{\psi+} \tilde{\beta}^{\ell^{+}} \quad, \quad{ }^{\psi+} \tilde{\mu}_{\eta}{ }^{u^{-}} \longrightarrow{ }^{\psi+} \tilde{\gamma}^{u^{-}} \quad, \quad{ }^{\psi+} \tilde{\mu}_{\eta}{ }^{u^{+}} \longrightarrow{ }^{\psi+} \tilde{\gamma}^{u^{+}}$, ${ }^{\psi+} \tilde{\mu}_{\eta}{ }^{u^{-}} \longrightarrow{ }^{\psi+} \tilde{\beta}^{u^{-}}$, and ${ }^{\psi+} \tilde{\mu}_{\eta}{ }^{u^{+}} \longrightarrow{ }^{\psi+} \tilde{\beta}^{u^{+}}$.

Theorem 2.5. Let $\left(\left[\tilde{Z}_{\eta}\right]\right) \subset\left[\tilde{\mathcal{F}}_{\neg N}^{* *}\right],[\widetilde{W}] \in\left[\tilde{\mathcal{F}}_{\neg N}^{* *}\right]$. Then $\left(\left[\tilde{Z}_{\eta}\right]\right)$ is $\left[\gamma^{\star}, \gamma^{\star \star}\right]$-level converges to $[\widetilde{W}]$ if and only if ${ }^{\left[\gamma^{\star}, \gamma^{\star \star}\right]+}\left[\tilde{\mu}_{\eta}\right] \longrightarrow{ }^{\left[\gamma^{\star}, \gamma^{\star \star}\right]+}[\tilde{\gamma}]$ and $\left[\gamma^{\star}, \gamma^{\star \star}\right]+\left[\tilde{\lambda}_{\eta}\right] \longrightarrow{ }^{\left[\gamma^{\star}, \gamma^{\star \star}\right]+}[\tilde{\beta}]$.

Proof: We have, ${ }^{\gamma^{\star}+} \tilde{\mu}_{\eta}^{l-} \mathrm{\vee}^{\gamma^{\star \star}+} \tilde{\mu}_{\eta}^{u-} \longrightarrow{ }^{\gamma^{\star}+} \tilde{\gamma}^{l^{-}} \mathrm{\vee}^{\gamma^{\star \star}+} \tilde{\gamma}^{u^{-}},{ }^{\gamma^{\star}+} \tilde{\mu}_{\eta}^{l+} \wedge^{\gamma^{\star \star}+} \tilde{\mu}_{\eta}^{u+}$ $\longrightarrow{ }^{\gamma^{\star}+} \tilde{\gamma}^{l^{+}} \wedge^{\gamma^{\star \star}+} \tilde{\gamma}^{u+}, \gamma^{\star}+\tilde{\lambda}_{\eta}^{l-} \vee^{\gamma^{\star \star}+} \tilde{\lambda}_{\eta}^{u^{-}} \longrightarrow{ }^{\gamma^{\star}+} \tilde{\beta}^{l^{-}} \vee^{\gamma^{\star \star}+} \tilde{\beta}^{u^{-}}$and ${ }^{\gamma^{\star}+} \tilde{\lambda}_{\eta}^{l+} \wedge$ $\gamma^{\star \star}+\tilde{\lambda}_{\eta}^{u^{+}} \longrightarrow{ }^{\gamma^{\star}+} \tilde{\beta}^{l^{+}} \wedge^{\gamma^{\star \star}+} \tilde{\beta}^{u^{+}}$, if and only if $\left[{ }^{\gamma^{\star}+} \tilde{\mu}_{\eta}^{l-} \vee^{\gamma^{\star \star}+} \tilde{\mu}_{\eta}^{u-}, \gamma^{\star}+\tilde{\mu}_{\eta}^{l+} \wedge\right.$ $\left.\gamma^{\star \star}+\tilde{\mu}_{\eta}^{u+}\right] \longrightarrow\left[{ }^{\gamma^{\star}+} \tilde{\gamma}^{l^{-}} \vee^{\gamma^{\star \star}+} \tilde{\gamma}^{u^{-}}, \gamma^{\star}+\tilde{\gamma}^{l^{+}} \wedge^{\gamma^{\star \star}+} \tilde{\gamma}^{u+}\right] \quad, \quad$ and $\quad\left[\gamma^{\gamma^{\star}+} \tilde{\lambda}_{\eta}^{l^{-}} \mathrm{\vee}\right.$ $\left.\gamma^{\star \star}+\tilde{\lambda}_{\eta}^{u^{-}}, \gamma^{\star}+\tilde{\lambda}_{\eta}^{l+} \wedge^{\gamma^{\star \star}+} \tilde{\lambda}_{\eta}^{u^{+}}\right] \longrightarrow\left[\gamma^{\star}+\tilde{\beta}^{l^{-}} \vee^{\gamma^{\star \star}+} \tilde{\beta}^{u^{-}}, \gamma^{\star}+\tilde{\beta}^{l^{+}} \wedge^{\gamma^{\star \star}+} \tilde{\beta}^{u^{+}}\right]$if and only if $\left[{ }^{\gamma^{\star}+} \tilde{\mu}_{\eta}^{l-}, \gamma^{\star}+\tilde{\mu}_{\eta}^{l+}\right] \cap\left[\gamma^{\gamma^{\star}+} \tilde{\mu}_{\eta}^{u-}, \gamma^{\star \star}+\tilde{\mu}_{\eta}^{u+}\right] \longrightarrow \quad\left[\gamma^{\star}+\tilde{\gamma}^{l^{-}}, \gamma^{\star}+\tilde{\gamma}^{l^{+}}\right] \cap$ $\left[\gamma^{\gamma^{\star}+} \tilde{\gamma}^{u-}, \gamma^{{ }^{\star \star}+} \tilde{\gamma}^{u+}\right] \quad$ and $\quad\left[\gamma^{\star}+\tilde{\lambda}_{\eta}^{l}{ }^{-}, \gamma^{\star}+\tilde{\lambda}_{\eta}^{l+}\right] \cap\left[\gamma^{\star \star}+\tilde{\lambda}_{\eta}^{u^{-}}, \gamma^{\star \star}+\tilde{\lambda}_{\eta}^{u}\right]$ $\longrightarrow\left[\gamma^{\star}+\tilde{\beta}^{l^{-}}, \gamma^{\star}+\tilde{\beta}^{l^{+}}\right] \cap\left[\gamma^{\star \star}+\tilde{\beta}^{u^{-}}, \gamma^{\star \star}+\tilde{\beta}^{u^{+}}\right] \quad$ if $\quad$ and $\quad$ only if $\gamma^{\star}+\tilde{\mu}_{\eta}^{l} \cap{ }^{\gamma^{\star \star}+} \tilde{\mu}_{\eta}^{u} \longrightarrow{ }^{\gamma^{\star}+} \tilde{\gamma}^{l} \cap{ }^{\gamma^{\star \star}+} \tilde{\gamma}^{u}$ and $\gamma^{\star}+\tilde{\lambda}_{\eta}^{l} \cap^{\gamma^{\star \star}+} \tilde{\lambda}_{\eta}^{u} \longrightarrow{ }^{\gamma^{\star}+} \tilde{\beta}^{l} \cap^{\gamma^{\star \star}+} \tilde{\beta}^{u}$. This completes the proof.

Theorem 2.6. Let $\left(\left[\widetilde{Z}_{\eta}\right]\right) \subset\left[\widetilde{\mathcal{F}}_{\neg N}^{* *}\right],[\widetilde{W}] \in\left[\tilde{\mathcal{F}}_{\neg N}^{* *}\right]$. Then $\left(\left[\widetilde{Z}_{\eta}\right]\right) \stackrel{\widetilde{\Gamma}}{\longrightarrow}[\widetilde{W}]$ if and only if $\left(\left[\tilde{\mu}_{\eta}\right]\right)$ and $\left(\left[\tilde{\lambda}_{\eta}\right]\right)$ are $\left[\gamma^{*}, \gamma^{*}\right]$-level converges to $[\tilde{\gamma}]$ and $[\tilde{\beta}]$, respectively. 
Proof: By hypothesis, we have $\left(\left[\tilde{Z}_{\eta}\right]\right) \stackrel{\left[\gamma^{\star}, \gamma^{\star}\right] \text {-level }}{\longrightarrow}[\widetilde{W}]$ if and only if ${ }^{\left[\gamma^{\star}, \gamma^{\star}\right]+}\left[\tilde{\mu}_{\eta}\right] \longrightarrow{ }^{\left[\gamma^{\star}, \gamma^{\star}\right]+}[\tilde{\gamma}]$ and ${ }^{\left[\gamma^{\star}, \gamma^{\star}\right]+}\left[\tilde{\lambda}_{\eta}\right] \longrightarrow{ }^{\left[\gamma^{\star}, \gamma^{\star}\right]+}[\tilde{\beta}]$ if and only if $\gamma^{\star}+\tilde{\mu}_{\eta}^{l} \cap{ }^{\gamma^{\star}+} \tilde{\mu}_{\eta}^{u} \longrightarrow{ }^{\gamma^{\star}+} \tilde{\gamma}^{l} \cap \gamma^{\star}+\tilde{\gamma}^{u}$ and $\gamma^{\star}+\tilde{\lambda}_{\eta}^{l} \cap^{\gamma^{\star}+} \tilde{\lambda}_{\eta}^{u} \longrightarrow \gamma^{\gamma^{*}+} \tilde{\beta}^{l} \cap^{\gamma^{\star}+} \tilde{\beta}^{u}$ if and only if $\left[\gamma^{\star}+\tilde{\mu}_{\eta}^{l-} \vee^{\gamma^{\star}+} \tilde{\mu}_{\eta}^{u-}, \gamma^{\star}+\tilde{\mu}_{\eta}^{l+} \wedge^{\gamma^{\star}+} \tilde{\mu}_{\eta}^{u+}\right] \longrightarrow\left[{ }^{\gamma^{\star}+} \tilde{\gamma}^{l^{-}} \vee^{\gamma^{\star}+} \tilde{\gamma}^{u^{-}}\right.$, $\left.\gamma^{\star}+\tilde{\gamma}^{l^{+}} \wedge^{\gamma^{\star}+} \tilde{\gamma}^{u^{+}}\right]$, and $\left[\gamma^{\gamma^{\star}} \tilde{\lambda}_{\eta}^{l^{-}} \vee^{\gamma^{\star}+} \tilde{\lambda}_{\eta}^{u^{-}}, \gamma^{\star}+\tilde{\lambda}_{\eta}^{l}{ }^{+} \wedge^{\gamma^{\star}+} \tilde{\lambda}_{\eta}^{u^{+}}\right] \longrightarrow\left[\gamma^{\gamma^{+}} \tilde{\beta}^{l^{-}} \vee\right.$ $\left.\gamma^{\star}+\tilde{\beta}^{u^{-}}, \gamma^{\star}+\tilde{\beta}^{l^{+}} \wedge^{\gamma^{\star}+} \tilde{\beta}^{u^{+}}\right]$, if and only if $\left(\left[\tilde{\mu}_{\eta}\right]\right) \stackrel{\left[\gamma^{\star}, \gamma^{\star}\right]-\text { level }}{\longrightarrow}[\tilde{\gamma}]$ and $\left(\left[\tilde{\lambda}_{\eta}\right]\right) \stackrel{\left[\gamma^{\star}, \gamma^{\star}\right]-\text { level }}{\longrightarrow}[\tilde{\beta}]$.

Theorem 2.7. Let $\left(\left[\tilde{Z}_{\eta}\right]=\left[\tilde{\mu}_{\eta}\right][+] i\left[\tilde{\gamma}_{\eta}\right]\right),\left(\left[\widetilde{W}_{\eta}\right]=\left[\tilde{\lambda}_{\eta}\right][+] i\left[\tilde{\beta}_{\eta}\right]\right),\left(\left[W_{\eta}^{\prime}\right]=\right.$ $\left.\left[\tilde{\delta}_{\eta}\right][+] i\left[\widetilde{\Delta}_{\eta}\right]\right) \subset\left[\tilde{\mathcal{F}}_{\neg N}^{* *}\right] \quad$ and $\quad[\tilde{Z}]=[\tilde{\mu}][+] i[\tilde{\gamma}],[\widetilde{W}]=[\tilde{\lambda}][+] i[\tilde{\beta}],\left[\widetilde{W}^{\prime}\right]=$ $[\tilde{\delta}][+] i[\widetilde{\Delta}] \in\left[\tilde{\mathcal{F}}_{\neg N}^{* *}\right], \quad[*] \in\{[+],[-],[\cdot],[/]\}$. If $\left(\left[\tilde{Z}_{\eta}\right]\right) \stackrel{\left[\gamma^{\star}, \gamma^{\star \star}\right]-\text { level }}{\longrightarrow}[\tilde{Z}]$ and $\left(\left[\widetilde{W}_{\eta}\right]\right) \stackrel{\left[\gamma^{\star}, \gamma^{\star \star}\right] \text {-level }}{\longrightarrow}[\widetilde{W}]$. Then

1. $\left(\left[\tilde{Z}_{\eta}\right]\right)[*]\left(\left[\widetilde{W}_{\eta}\right]\right) \stackrel{\left[\gamma^{\star}, \gamma^{\star \star}\right]-\text { level }}{\longrightarrow}[\tilde{Z}][*][\widetilde{W}]$, and $[\tilde{Z}]=[\widetilde{W}]$ where $\left(\left[\tilde{Z}_{\eta}\right]\right)$ is also $\left[\gamma^{\star}, \gamma^{\star \star}\right]$-level converges to $[\widetilde{W}]$.

2. $\left(\left[\widetilde{W}_{\eta}^{\prime}\right]\right) \stackrel{\left[\gamma^{\star}, \gamma^{\star \star}\right] \text {-level }}{\longrightarrow}[\widetilde{W}]$ where $[\tilde{Z}]=[\widetilde{W}],{ }^{\left[\gamma^{\star}, \gamma^{\star \star}\right]+}\left[\tilde{\mu}_{\eta}\right] \leq^{\left[\gamma^{\star}, \gamma^{\star \star}\right]+}\left[\tilde{\delta}_{\eta}\right]$, $\left[\gamma^{\star}, \gamma^{\star \star}\right]+\left[\tilde{\gamma}_{\eta}\right] \leq{ }^{\left[\gamma^{\star}, \gamma^{\star \star}\right]+}\left[\widetilde{\Delta}_{\eta}\right], \quad{ }^{\left[\gamma^{\star}, \gamma^{\star \star}\right]+}\left[\tilde{\delta}_{\eta}\right] \leq{ }^{\left[\gamma^{\star}, \gamma^{\star \star}\right]+}\left[\tilde{\lambda}_{\eta}\right], \quad$ and ${ }^{\left[\gamma^{\star}, \gamma^{\star \star}\right]+}\left[\widetilde{\Delta}_{\eta}\right] \leq$ $\left[\gamma^{\star}, \gamma^{\star \star}\right]+\left[\tilde{\beta}_{\eta}\right]$. Also, ${ }^{\left[\gamma^{\star}, \gamma^{\star \star}\right]+}[\tilde{\gamma}] \leq{ }^{\left[\gamma^{\star}, \gamma^{\star \star}\right]+}[\tilde{\Delta}]$ and ${ }^{\left[\gamma^{\star}, \gamma^{\star \star}\right]+}[\tilde{\mu}] \leq^{\left[\gamma^{\star}, \gamma^{\star \star}\right]+}[\tilde{\delta}]$ where $\left[\gamma^{\star}, \gamma^{\star \star}\right]+\left[\tilde{\mu}_{\eta}\right] \leq^{\left[\gamma^{\star}, \gamma^{\star \star}\right]+}[\tilde{\delta}]$ and ${ }^{\left[\gamma^{\star}, \gamma^{\star \star}\right]+}\left[\tilde{\gamma}_{\eta}\right] \leq^{\left[\gamma^{\star}, \gamma^{\star \star}\right]+}[\widetilde{\Delta}]$.

Proof: We only prove (1). By hypothesis, we have ${ }^{\left[\gamma^{\star}, \gamma^{\star \star}\right]+}\left[\tilde{\mu}_{\eta}\right] \longrightarrow{ }^{\left[\gamma^{\star}, \gamma^{\star \star}\right]+}[\tilde{\mu}]$, $\left[\gamma^{\star}, \gamma^{\star \star}\right]+\left[\tilde{\gamma}_{\eta}\right] \longrightarrow{ }^{\left[\gamma^{\star}, \gamma^{\star \star}\right]+}[\tilde{\gamma}],{ }^{\left[\gamma^{\star}, \gamma^{\star \star}\right]+}\left[\tilde{\lambda}_{\eta}\right] \longrightarrow$ ${ }^{\left[\gamma^{\star}, \gamma^{\star \star}\right]+}[\tilde{\beta}],{ }^{\left[\gamma^{\star}, \gamma^{\star \star}\right]+}\left[\tilde{\delta}_{\eta}\right] \longrightarrow{ }^{\left[\gamma^{\star}, \gamma^{\star \star}\right]+}[\tilde{\delta}],{ }^{\left[\gamma^{\star}, \gamma^{\star \star}\right]+}\left[\widetilde{\Delta}_{\eta}\right] \longrightarrow{ }^{\left[\gamma^{\star}, \gamma^{\star \star}\right]+}[\widetilde{\Delta}]$. Also,

$\left[\gamma^{\star}, \gamma^{\star \star}\right]+\left(\left[\tilde{Z}_{\eta}\right][*]\left[\widetilde{W}_{\eta}\right]\right)={ }^{\left[\gamma^{\star}, \gamma^{\star \star}\right]+}\left(\left[\tilde{\tilde{Z}}_{\eta}, \overline{\widetilde{Z}}_{\eta}\right]\right) *^{\left[\gamma^{\star}, \gamma^{\star \star}\right]+}\left(\left[\widetilde{W}_{\eta}, \overline{\widetilde{W}}_{\eta}\right]\right)$ 


$$
={ }^{\left[\gamma^{\star}, \gamma^{\star \star}\right]+}\left(\left[\widetilde{Z}_{\eta}\right]\right) *{ }^{\left[\gamma^{\star}, \gamma^{\star \star}\right]+}\left(\left[\widetilde{W}_{\eta}\right]\right), \text { and }
$$

$\left.\left[\gamma^{\star}, \gamma^{\star \star}\right]+([\tilde{Z}]) *{ }^{\left[\gamma^{\star}, \gamma^{\star \star}\right]+}([\widetilde{W}])\right)^{\left[\gamma^{\star}, \gamma^{\star \star}\right]+}([\underline{\tilde{Z}}, \overline{\tilde{Z}}]) *{ }^{\left[\gamma^{\star}, \gamma^{\star \star}\right]+}([\tilde{\widetilde{W}}, \overline{\widetilde{W}}])$

$$
=^{\left[\gamma^{\star}, \gamma^{\star \star}\right]+}([\underline{\tilde{Z}}, \overline{\tilde{Z}}][*][\underline{\widetilde{W}}, \overline{\widetilde{W}}])
$$

The other part comes from ${ }^{\left[\gamma^{\star}, \gamma^{\star \star}\right]+}[\tilde{\mu}]={ }^{\left[\gamma^{\star}, \gamma^{\star \star}\right]+}[\tilde{\lambda}]$ and ${ }^{\left[\gamma^{\star}, \gamma^{\star \star}\right]+}[\tilde{\gamma}]={ }^{\left[\gamma^{\star}, \gamma^{\star \star}\right]+}[\tilde{\beta}]$, owing to, ${ }^{\left[\gamma^{\star}, \gamma^{\star \star}\right]+}\left[\tilde{\mu}_{\eta}\right] \longrightarrow{ }^{\left[\gamma^{\star}, \gamma^{\star \star}\right]+}[\tilde{\mu}] \quad, \quad{ }^{\left[\gamma^{\star}, \gamma^{\star \star}\right]+}\left[\tilde{\gamma}_{\eta}\right] \longrightarrow{ }^{\left[\gamma^{\star}, \gamma^{\star \star}\right]+}[\tilde{\gamma}] \quad$, $\left[\gamma^{\star}, \gamma^{\star \star}\right]+\left[\tilde{\mu}_{\eta}\right] \longrightarrow{ }^{\left[\gamma^{\star}, \gamma^{\star \star}\right]+}[\tilde{\lambda}]$, and ${ }^{\left[\gamma^{\star}, \gamma^{\star \star}\right]+}\left[\tilde{\gamma}_{\eta}\right] \longrightarrow{ }^{\left[\gamma^{\star}, \gamma^{\star \star}\right]+}[\tilde{\beta}]$.

Theorem 2.8. GRVBCCCNFNS $\widetilde{\sum_{\eta=1}^{\infty}}\left[\tilde{Z}_{\eta}\right]$ converges to GRVBCCCNFN $[\tilde{Z}]$ if and only if for any $\left(\gamma^{\star}, \gamma^{\star \star}\right) \in I_{[0}^{1[} \times I_{[0}^{1[}, \sum_{\eta=1}^{\infty}{ }^{\left[\gamma^{\star}, \gamma^{\star \star}\right]+}\left[\tilde{\mu}_{\eta}\right]={ }^{\left[\gamma^{\star}, \gamma^{\star \star}\right]+}[\tilde{\mu}]$ and $\sum_{\eta=1}^{\infty}{ }^{\left[\gamma^{\star}, \gamma^{\star \star}\right]+}\left[\tilde{\gamma}_{\eta}\right]={ }^{\left[\gamma^{\star}, \gamma^{\star \star}\right]+}[\tilde{\gamma}]$.

Proof: By hypothesis, we have $\left(\left[\tilde{\beta}_{\eta}\right]\right) \stackrel{\left[\gamma^{\star}, \gamma^{\star \star}\right]-\text { level }}{\longrightarrow}[\tilde{Z}]$ if and only if ${ }^{\left[\gamma^{\star}, \gamma^{\star \star}\right]+}\left(\widetilde{\sum_{n=1}^{\eta}}\left[\tilde{\mu}_{n}\right]\right) \longrightarrow{ }^{\left[\gamma^{\star}, \gamma^{\star \star}\right]+}[\tilde{\mu}]$ and ${ }^{\left[\gamma^{\star}, \gamma^{\star \star}\right]+}\left(\widetilde{\sum_{n=1}^{\eta}}\left[\tilde{\gamma}_{n}\right]\right) \longrightarrow{ }^{\left[\gamma^{\star}, \gamma^{\star \star}\right]+}[\tilde{\gamma}]$ if and only if $\sum_{n=1}^{\eta}{ }^{\left[\gamma^{\star}, \gamma^{\star \star}\right]+}\left[\tilde{\mu}_{n}\right] \longrightarrow{ }^{\left[\gamma^{\star}, \gamma^{\star \star}\right]+}[\tilde{\mu}]$ and $\sum_{n=1}^{\eta}{ }^{\left[\gamma^{\star}, \gamma^{\star \star}\right]+}\left[\tilde{\gamma}_{n}\right] \longrightarrow$ $\left[\gamma^{\star}, \gamma^{\star \star}\right]+[\tilde{\gamma}]$ if and only if ${ }^{\left[\gamma^{\star}, \gamma^{\star \star}\right]+}\left[\tilde{\mu}_{\eta}\right]$ and ${ }^{\left[\gamma^{\star}, \gamma^{\star \star}\right]+}\left[\tilde{\gamma}_{\eta}\right]$ are sequences of $\sum_{\eta=1}^{\infty}\left[\gamma^{\star}, \gamma^{\star \star}\right]+\left[\tilde{\mu}_{\eta}\right]$ and $\sum_{\eta=1}^{\infty}{ }^{\left[\gamma^{\star}, \gamma^{\star \star}\right]+}\left[\tilde{\gamma}_{\eta}\right]$ respectively, if and only if $\sum_{\eta=1}^{\infty}\left[\gamma^{\star}, \gamma^{\star \star}\right]+\left[\tilde{\mu}_{\eta}\right]={ }^{\left[\gamma^{\star}, \gamma^{\star \star}\right]+}[\tilde{\mu}]$ and $\sum_{\eta=1}^{\infty}{ }^{\left[\gamma^{\star}, \gamma^{\star \star}\right]+}\left[\tilde{\gamma}_{\eta}\right]={ }^{\left[\gamma^{\star}, \gamma^{\star \star}\right]+}[\tilde{\gamma}]$.

Corollary 2.9. GRVBCCCNFNS $\widetilde{\sum_{\eta=1}^{\infty}}\left[\tilde{Z}_{\eta}\right]$ converges to GRVBCCCNFN $[\tilde{Z}]$ if and only if for any $\left(\gamma^{\star}, \gamma^{\star \star}\right) \in I_{[0}^{1[} \times I_{[0}^{1[}, \sum_{\eta=1}^{\infty}{ }^{\left[\gamma^{\star}, \gamma^{\star \star}\right]+}\left[\tilde{Z}_{\eta}\right]={ }^{\left[\gamma^{\star}, \gamma^{\star \star}\right]+}[\tilde{Z}]$.

Proof: The proof follows quickly from Theorem 2.8. 
Theorem 2.10. GRVBCCCNFNS $\widetilde{\sum_{\eta=1}^{\infty}}\left[\tilde{Z}_{\eta}\right]=\widetilde{\sum_{\eta=1}^{\infty}}\left[\tilde{\lambda}_{\eta}\right][+] i \widetilde{\sum_{\eta=1}^{\infty}}\left[\tilde{\varphi}_{\eta}\right]$ converges to GRVBCCCNFN $[\tilde{Z}]=[\tilde{\lambda}][+] i[\tilde{\varphi}]$ if and only if $\sum_{\eta=1}^{\infty} \gamma^{\star}+\tilde{\lambda}_{\eta}^{\ell}=\gamma^{\star}+\tilde{\lambda}^{\ell}$, $\sum_{\eta=1}^{\infty}{ }^{\gamma^{\star}+} \tilde{\varphi}_{\eta}^{\ell}={ }^{\gamma^{\star}+} \tilde{\varphi}^{\ell}, \sum_{\eta=1}^{\infty}{ }^{\star \star}+\tilde{\lambda}_{\eta}^{u}={ }^{\gamma^{\star \star}}+\tilde{\lambda}^{u}$ and $\sum_{\eta=1}^{\infty}{ }^{\gamma^{\star \star}+} \tilde{\varphi}_{\eta}^{u}={ }^{\gamma^{\star \star}+} \tilde{\varphi}^{u}$.

Proof: We have $\left(\left[\tilde{\beta}_{\eta}\right]\right) \stackrel{\left[\gamma^{\star}, \gamma^{\star \star}\right] \text {-level }}{\longrightarrow}[\tilde{Z}]=[\tilde{\lambda}][+] i[\tilde{\varphi}]$ if and only if $\left(\left[\tilde{\lambda}_{\eta}\right]\right) \stackrel{\left[\gamma^{\star}, \gamma^{\star \star}\right]-\text { level }}{\longrightarrow}[\tilde{\lambda}]$ and $\left(\left[\tilde{\varphi}_{\eta}\right]\right) \stackrel{\left[\gamma^{\star}, \gamma^{\star \star}\right] \text {-level }}{\longrightarrow}[\tilde{\varphi}]$ if and only if $\tilde{\lambda}_{\eta}^{\ell} \stackrel{\gamma^{\star}-\text { level }}{\longrightarrow} \tilde{\lambda}^{\ell}$, $\tilde{\lambda}_{\eta}^{u} \stackrel{\gamma^{\star \star}-\text { level }}{\longrightarrow} \tilde{\lambda}^{u}, \tilde{\varphi}_{\eta}^{\ell} \stackrel{\gamma^{\star}-\text { level }}{\longrightarrow} \tilde{\varphi}^{\ell}$, and $\tilde{\varphi}_{\eta}^{u} \stackrel{\gamma^{\star \star}-\text { level }}{\longrightarrow} \tilde{\varphi}^{u}$.

Theorem 2.11. GRVBCCCNFNS $\widetilde{\sum_{\eta=1}^{\infty}}\left[\tilde{Z}_{\eta}\right]$ converges to GRVBCCCNFN $[\tilde{Z}]$ if and only if $\sum_{\eta=1}^{\infty} \gamma^{\star}+\tilde{\lambda}_{\eta}^{\ell^{-}}={ }^{\gamma^{\star}+} \tilde{\lambda}^{\ell^{-}}, \sum_{\eta=1}^{\infty} \gamma^{\star}+\tilde{\lambda}_{\eta}^{\ell^{+}}=\gamma^{\star}+\tilde{\lambda}^{\ell^{+}}, \sum_{\eta=1}^{\infty} \gamma^{\star}+\tilde{\varphi}_{\eta}^{\ell^{-}}=$ $\gamma^{\star}+\tilde{\varphi}^{\ell^{-}}, \quad \sum_{\eta=1}^{\infty}{ }^{\gamma^{\star}+} \tilde{\varphi}_{\eta}^{\ell^{+}}={ }^{\gamma^{\star}+} \tilde{\varphi}^{\ell^{+}}, \quad \sum_{\eta=1}^{\infty}{ }^{\gamma^{\star}+} \tilde{\lambda}_{\eta}^{u^{-}}={ }^{\gamma^{\star \star}+} \tilde{\lambda}^{u^{-}}, \sum_{\eta=1}^{\infty}{ }^{\gamma^{\star \star}+} \tilde{\lambda}_{\eta}^{u^{+}}=$ $\gamma^{\star \star}+\tilde{\lambda}^{u^{+}}, \sum_{\eta=1}^{\infty}{ }^{\gamma^{\star \star}+} \tilde{\varphi}_{\eta}^{u^{-}}={ }^{\gamma^{\star \star}+} \tilde{\varphi}^{u^{-}}$and $\sum_{\eta=1}^{\infty}{ }^{\gamma^{\star \star}+} \tilde{\varphi}_{\eta}^{u+}={ }^{\gamma^{\star \star}+} \tilde{\varphi}^{u^{+}}$.

Proof: $\left(\left[\tilde{\lambda}_{\eta}\right]\right) \stackrel{\left[\gamma^{\star}, \gamma^{\star \star}\right] \text {-level }}{\longrightarrow}[\tilde{\lambda}]$ and $\left(\left[\tilde{\varphi}_{\eta}\right]\right) \stackrel{\left[\gamma^{\star}, \gamma^{\star \star}\right] \text {-level }}{\longrightarrow}[\tilde{\varphi}]$ if and only if $\tilde{\lambda}_{\eta}^{\ell} \stackrel{\gamma^{\star}-\text { level }}{\longrightarrow} \tilde{\lambda}^{\ell}, \tilde{\lambda}_{\eta}^{u} \stackrel{\gamma^{\star \star}-\text { level }}{\longrightarrow} \tilde{\lambda}^{u}, \tilde{\varphi}_{\eta}^{\ell} \stackrel{\gamma^{\star}-\text { level }}{\longrightarrow} \tilde{\varphi}^{\ell}$, and $\tilde{\varphi}_{\eta}^{u} \stackrel{\gamma^{\star \star}-\text { level }}{\longrightarrow} \tilde{\varphi}^{u}$ if and only if $\gamma^{\star}+\tilde{\lambda}_{\eta}^{\ell^{-}} \longrightarrow \gamma^{\gamma^{*}+} \tilde{\lambda}^{\ell^{-}}, \quad \gamma^{\star}+\tilde{\lambda}_{\eta}^{\ell^{+}} \longrightarrow \gamma^{\gamma^{*}+} \tilde{\lambda}^{\ell^{+}}, \quad \gamma^{\star}+\tilde{\varphi}_{\eta}^{\ell^{-}} \longrightarrow \gamma^{\gamma^{*}} \tilde{\varphi}^{\ell^{-}}$, $\gamma^{\star}+\tilde{\varphi}_{\eta}^{\ell^{+}} \longrightarrow{ }^{\gamma^{\star}+} \tilde{\varphi}^{\ell^{+}}, \quad \gamma^{\star \star}+\tilde{\lambda}_{\eta}^{u^{-}} \longrightarrow{ }^{\gamma^{\star \star}}+\tilde{\lambda}^{u^{-}}, \quad \gamma^{\star \star}+\tilde{\lambda}_{\eta}^{u^{+}} \longrightarrow{ }^{\gamma^{\star \star}+} \tilde{\lambda}^{u^{+}}$, $\gamma^{\star \star}+\tilde{\varphi}_{\eta}^{u-} \longrightarrow{ }^{\gamma^{\star \star}}+\tilde{\varphi}^{u^{-}}$and ${ }^{\gamma^{\star \star}+} \tilde{\varphi}_{\eta}^{u^{+}} \longrightarrow{ }^{\gamma^{\star \star}+} \tilde{\varphi}^{u^{+}}$if and only if $\sum_{n=1}^{\eta}{ }^{\gamma^{\star}+} \tilde{\lambda}_{n}^{\ell^{-}}=$ $\gamma^{\star}+\tilde{\lambda}^{\ell^{-}}, \sum_{n=1}^{\eta} \gamma^{\star}+\tilde{\lambda}_{n}^{\ell^{+}}=\gamma^{\star}+\tilde{\lambda}^{\ell^{+}}, \sum_{n=1}^{\eta} \gamma^{\star}+\tilde{\varphi}_{n}^{\ell^{-}}=\gamma^{\star}+\tilde{\varphi}^{\ell^{-}}, \sum_{n=1}^{\eta} \gamma^{\star}+\tilde{\varphi}_{n}^{\ell^{+}}=\gamma^{\star}+\tilde{\varphi}^{\ell^{+}}$, $\sum_{n=1}^{\eta}{ }^{\gamma^{\star \star}}+\tilde{\lambda}_{n}^{u^{-}}={ }^{\gamma^{\star \star}+} \tilde{\lambda}^{u^{-}}, \sum_{n=1}^{\eta}{ }^{\gamma^{\star \star}+} \tilde{\lambda}_{n}^{u^{+}}={ }^{\gamma^{\star \star}+} \tilde{\lambda}^{u^{+}}, \sum_{n=1}^{\eta}{ }^{\gamma^{\star \star}+} \tilde{\varphi}_{n}^{u^{-}}={ }^{\gamma^{\star \star}+} \tilde{\varphi}^{u^{-}}$and $\sum_{n=1}^{\eta}{ }^{\gamma^{\star}+} \tilde{\varphi}_{n}^{u+}={ }^{\gamma^{\star \star}+} \tilde{\varphi}^{u+}$

Theorem 2.12. For GRVBCCCNFN $[\tilde{Z}]$ and $[\widetilde{W}]$ if GRVBCCCNFNS $\widetilde{\sum_{\eta=1}^{\infty}}\left[\tilde{Z}_{\eta}\right]$ converge to $[\tilde{Z}]$. Then $\widetilde{\sum_{\eta=1}^{\infty}}\left(\left[\tilde{Z}_{\eta}\right][\cdot][\widetilde{W}]\right)=[\widetilde{W}][\cdot] \widetilde{\sum_{\eta=1}^{\infty}}\left[\tilde{Z}_{\eta}\right]$.

Proof: Since GRVBCCCNFNS $\widetilde{\sum_{\eta=1}^{\infty}}\left[\tilde{Z}_{\eta}\right]$ converges to GRVBCCCNFN [Z̈], we have $\sum_{\eta=1}^{\infty}\left[\gamma^{\star}, \gamma^{\star \star}\right]+\left[\tilde{Z}_{\eta}\right]={ }^{\left[\gamma^{\star}, \gamma^{\star \star}\right]+}[\tilde{Z}]$. So that,

$$
\sum_{\eta=1}^{\infty}{ }^{\left[\gamma^{\star}, \gamma^{\star \star}\right]+}\left(\left[\tilde{Z}_{\eta}\right][\cdot][\widetilde{W}]\right)=\sum_{\eta=1}^{\infty}\left({ }^{\left[\gamma^{\star}, \gamma^{\star \star}\right]+}\left[\tilde{Z}_{\eta}\right] \cdot{ }^{\left[\gamma^{\star}, \gamma^{\star \star}\right]+}[\widetilde{W}]\right)
$$




$$
\begin{aligned}
& ={ }^{\left[\gamma^{\star}, \gamma^{\star \star}\right]+}[\widetilde{W}] \cdot \sum_{n=1}^{\infty}\left[\gamma^{\star}, \gamma^{\star \star}\right]+\left[\tilde{Z}_{\eta}\right] \\
& ={ }^{\left[\gamma^{\star}, \gamma^{\star \star}\right]+}[\widetilde{W}] \cdot\left[\gamma^{\star}, \gamma^{\star \star}\right]+[\tilde{Z}]
\end{aligned}
$$

Theorem 2.13. Let GRVBCCCNFNS $\widetilde{\sum_{\eta=1}^{\infty}}\left[\tilde{Z}_{\eta}\right]$ and $\widetilde{\sum_{\eta=1}^{\infty}}\left[\widetilde{W}_{\eta}\right]$ converge to GRVBCCCNFN $[\tilde{Z}]$ and $[\widetilde{W}]$, respectively. Then for the extended basic arithmetic operation $[*]$, we have $\widetilde{\sum_{\eta=1}^{\infty}}\left(\left[\tilde{Z}_{\eta}\right][*]\left[\widetilde{W}_{\eta}\right]\right)=\widetilde{\sum_{\eta=1}^{\infty}}\left[\tilde{Z}_{\eta}\right][*] \widetilde{\sum_{\eta=1}^{\infty}}\left[\widetilde{W}_{\eta}\right]$.

Proof: We only prove the case of extended addition. Suppose that $\left[\tilde{Z}_{\eta}\right]=$ $\left[\tilde{\lambda}_{\eta}\right][+] i\left[\tilde{\varphi}_{\eta}\right],\left[\widetilde{W}_{\eta}\right]=\left[\tilde{\gamma}_{\eta}\right][+] i\left[\tilde{\delta}_{\eta}\right],[\tilde{Z}]=[\tilde{\lambda}][+] i[\tilde{\varphi}]$ and $[\tilde{W}]=[\tilde{\gamma}][+] i[\tilde{\delta}] . \mathrm{We}$ have

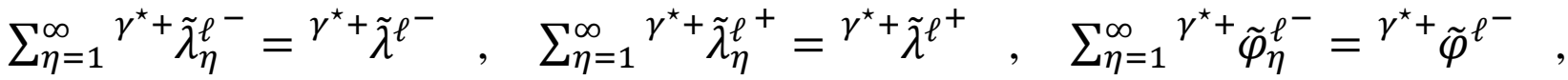
$\sum_{\eta=1}^{\infty}{ }^{\gamma^{\star}+} \tilde{\varphi}_{\eta}^{\ell^{+}}={ }^{\gamma^{\star}+} \tilde{\varphi}^{\ell^{+}}, \sum_{\eta=1}^{\infty}{ }^{{ }^{\star}}+\tilde{\lambda}_{\eta}^{u^{-}}={ }^{\gamma^{\star \star}+} \tilde{\lambda}^{u^{-}}, \quad \sum_{\eta=1}^{\infty}{ }^{{ }^{\star \star}}+\tilde{\lambda}_{\eta}^{u^{+}}={ }^{\gamma^{\star \star}+} \tilde{\lambda}^{u^{+}}$, $\sum_{\eta=1}^{\infty}{ }^{{ }^{\star}+} \tilde{\varphi}_{\eta}^{u^{-}}={ }^{\gamma^{\star \star}+} \tilde{\varphi}^{u^{-}}$and $\sum_{\eta=1}^{\infty}{ }^{\star \star}+\tilde{\varphi}_{\eta}^{u+}={ }^{\gamma^{\star \star}+} \tilde{\varphi}^{u^{+}}$. Also, $\sum_{\eta=1}^{\infty}{ }^{\gamma^{\star}+} \tilde{\gamma}_{\eta}^{\ell^{-}}=$ $\gamma^{\star}+\tilde{\gamma}^{\ell^{-}}, \sum_{\eta=1}^{\infty}{ }^{\gamma^{\star}} \tilde{\gamma}_{\eta}^{\ell^{+}}=\gamma^{\star}+\tilde{\gamma}^{\ell^{+}}, \sum_{\eta=1}^{\infty} \gamma^{\star}+\tilde{\delta}_{\eta}^{\ell^{-}}=\gamma^{\star}+\tilde{\delta}^{\ell^{-}}, \sum_{\eta=1}^{\infty} \gamma^{\star}+\tilde{\delta}_{\eta}^{\ell^{+}}=\gamma^{\star}+\tilde{\delta}^{\ell^{+}}$, $\sum_{\eta=1}^{\infty}{ }^{\gamma^{\star}+} \tilde{\gamma}_{\eta}^{u^{-}}={ }^{\gamma^{\star \star}+} \tilde{\gamma}^{u^{-}}, \sum_{\eta=1}^{\infty}{ }^{\gamma^{\star}+} \tilde{\gamma}_{\eta}^{u^{+}}={ }^{\gamma^{\star \star}}+\tilde{\gamma}^{u^{+}}, \sum_{\eta=1}^{\infty}{ }^{\gamma^{\star \star}+} \tilde{\delta}_{\eta}^{u^{-}}={ }^{\gamma^{\star \star}+} \tilde{\delta}^{u^{-}}$and $\sum_{\eta=1}^{\infty}{ }^{{ }^{\star}+} \tilde{\delta}_{\eta}^{u+}=\gamma^{\star \star}+\tilde{\delta}^{u^{+}}$. So that,

$\sum_{\eta=1}^{\infty} \gamma^{\star}+\tilde{\lambda}_{\eta}^{\ell^{-}}+\sum_{\eta=1}^{\infty} \gamma^{\star}+\tilde{\gamma}_{\eta}^{\ell^{-}}=\gamma^{\star}+\tilde{\lambda}^{\ell^{-}}+{ }^{\gamma^{\star}+} \tilde{\gamma}^{\ell^{-}}, \sum_{\eta=1}^{\infty} \gamma^{\star}+\tilde{\lambda}_{\eta}^{\ell^{+}}+\sum_{\eta=1}^{\infty}{ }^{\gamma^{*}+} \tilde{\gamma}_{\eta}^{\ell^{+}}=$ $\gamma^{\star}+\tilde{\lambda}^{\ell^{+}}+{ }^{\gamma^{\star}} \tilde{\gamma}^{\ell^{+}} \quad, \quad \sum_{\eta=1}^{\infty}{ }^{\gamma^{\star}+} \tilde{\varphi}_{\eta}^{\ell^{-}}+\sum_{\eta=1}^{\infty} \gamma^{\star}+\tilde{\delta}_{\eta}^{\ell^{-}}={ }^{\gamma^{\star}+} \tilde{\varphi}^{\ell^{-}}+{ }^{\gamma^{\star}+} \tilde{\delta}^{\ell^{-}}$ $\sum_{\eta=1}^{\infty} \gamma^{\star}+\tilde{\varphi}_{\eta}^{\ell^{+}}+\sum_{\eta=1}^{\infty} \gamma^{\star}+\tilde{\delta}_{\eta}^{\ell^{+}}=\gamma^{\star}+\tilde{\varphi}^{\ell^{+}}+{ }^{\gamma^{\star}}+\tilde{\delta}^{\ell^{+}}$

$\sum_{\eta=1}^{\infty} \gamma^{\star \star}+\tilde{\lambda}_{\eta}^{u^{-}}+\sum_{\eta=1}^{\infty}{ }^{{ }^{\star \star}+} \tilde{\gamma}_{\eta}^{u^{-}}={ }^{\gamma^{\star \star}}+\tilde{\lambda}^{u^{-}}+{ }^{\gamma^{\star \star}+} \tilde{\gamma}^{u^{-}} \quad, \quad \sum_{\eta=1}^{\infty}{ }^{{ }^{\star \star}}+\tilde{\lambda}_{\eta}^{u^{+}}+$ $\sum_{\eta=1}^{\infty}{ }^{\gamma^{\star \star}}+\tilde{\gamma}_{\eta}^{u+}={ }^{\gamma^{\star \star}+} \tilde{\lambda}^{u^{+}}+{ }^{\gamma^{\star \star}+} \tilde{\gamma}^{u^{+}}, \sum_{\eta=1}^{\infty}{ }^{\gamma^{\star \star}}+\tilde{\varphi}_{\eta}^{u^{-}}+\sum_{\eta=1}^{\infty}{ }^{\gamma^{\star \star}}+\tilde{\delta}_{\eta}^{u^{-}}={ }^{\gamma^{\star \star}+} \tilde{\varphi}^{u^{-}}+$ $\gamma^{{ }^{\star}+} \tilde{\delta}^{u^{-}}$and $\sum_{\eta=1}^{\infty}{ }^{\gamma^{\star \star}+} \tilde{\varphi}_{\eta}^{u^{+}}+\sum_{\eta=1}^{\infty}{ }^{\gamma^{\star \star}}+\tilde{\delta}_{\eta}^{u^{+}}={ }^{\gamma^{\star \star}+} \tilde{\varphi}^{u^{+}}+{ }^{\gamma^{\star \star}+} \tilde{\delta}^{u^{+}}$. This implies that

$$
\begin{aligned}
& \sum_{\eta=1}^{\infty}\left({ }^{\gamma^{\star}+} \tilde{\lambda}_{\eta}^{\ell^{-}}+{ }^{\gamma^{\star}+} \tilde{\gamma}_{\eta}^{\ell^{-}}\right)={ }^{\gamma^{\star}+} \tilde{\lambda}^{\ell^{-}}+{ }^{\gamma^{\star}+} \tilde{\gamma}^{\ell^{-}} \quad, \quad \sum_{\eta=1}^{\infty}\left({ }^{\gamma^{\star}+} \tilde{\lambda}_{\eta}^{\ell^{+}}+{ }^{\gamma^{\star}+} \tilde{\gamma}_{\eta}^{\ell^{+}}\right)= \\
& \gamma^{\star}+\tilde{\lambda}^{\ell^{+}}+{ }^{\gamma^{\star}+} \tilde{\gamma}^{\ell^{+}}, \sum_{\eta=1}^{\infty}\left({ }^{\gamma^{\star}+} \tilde{\varphi}_{\eta}^{\ell^{-}}+{ }^{\gamma^{\star}+} \tilde{\delta}_{\eta}^{\ell^{-}}\right)={ }^{\gamma^{\star}+} \tilde{\varphi}^{\ell^{-}}+{ }^{\gamma^{\star}+} \tilde{\delta}^{\ell^{-}}, \sum_{\eta=1}^{\infty}\left({ }^{\gamma^{\star}+} \tilde{\varphi}_{\eta}^{\ell^{+}}+\right. \\
& \left.\gamma^{\star}+\tilde{\delta}_{\eta}^{\ell^{+}}\right)={ }^{\gamma^{\star}+} \tilde{\varphi}^{\ell^{+}}+{ }^{\gamma^{\star}+} \tilde{\delta}^{\ell^{+}}, \sum_{\eta=1}^{\infty}\left({ }^{\gamma^{\star \star}+} \tilde{\lambda}_{\eta}^{u^{-}}+{ }^{\gamma^{\star \star}+} \tilde{\gamma}_{\eta}^{u-}\right)={ }^{\gamma^{\star \star}+} \tilde{\lambda}^{u^{-}}+{ }^{\gamma^{\star \star}+} \tilde{\gamma}^{u-},
\end{aligned}
$$




$$
\begin{aligned}
& \sum_{\eta=1}^{\infty}\left({ }^{\star \star}+\tilde{\lambda}_{\eta}^{u^{+}}+{ }^{\gamma^{\star \star}+} \tilde{\gamma}_{\eta}^{u+}\right)={ }^{\gamma^{\star \star}}+\tilde{\lambda}^{u^{+}}+{ }^{\gamma^{\star \star}+} \tilde{\gamma}^{u+}, \quad \sum_{\eta=1}^{\infty}\left({ }^{\star \star}+\tilde{\varphi}_{\eta}^{u^{-}}+{ }^{\gamma^{\star \star}+} \tilde{\delta}_{\eta}^{u^{-}}\right)= \\
& { }^{\gamma^{\star \star}+} \tilde{\varphi}^{u^{-}}+{ }^{\gamma^{\star \star}+} \tilde{\delta}^{u^{-}} \text {and } \sum_{\eta=1}^{\infty}\left({ }^{\gamma^{\star \star}+} \tilde{\varphi}_{\eta}^{u+}+{ }^{\gamma^{\star \star}}+\tilde{\delta}_{\eta}^{u}\right)={ }^{\gamma^{\star \star}+} \tilde{\varphi}^{u^{+}}+{ }^{\gamma^{\star \star}+} \tilde{\delta}^{u^{+}} \text {. Hence } \\
& \sum_{\eta=1}^{\infty}{ }^{\gamma^{\star}+}\left(\tilde{\lambda}_{\eta}+\tilde{\gamma}_{\eta}\right)^{\ell^{-}}={ }^{\gamma^{\star}+}(\tilde{\lambda}+\tilde{\gamma})^{\ell^{-}}, \sum_{\eta=1}^{\infty}{ }^{\gamma^{\star}+}\left(\tilde{\lambda}_{\eta}+\tilde{\gamma}_{\eta}\right)^{\ell^{+}}={ }^{\gamma^{\star}+}(\tilde{\lambda}+\tilde{\gamma})^{\ell^{+}} \text {, } \\
& \sum_{\eta=1}^{\infty}{ }^{\gamma^{*}+}\left(\tilde{\gamma}_{\eta}+\tilde{\delta}_{\eta}\right)^{\ell^{-}}={ }^{\gamma^{\star}+}(\tilde{\varphi}+\tilde{\delta})^{\ell^{-}} \\
& \sum_{\eta=1}^{\infty}{ }^{\gamma^{\star}+}\left(\tilde{\varphi}_{\eta}+\tilde{\delta}_{\eta}\right)^{\ell^{+}}={ }^{\gamma^{\star}+}(\tilde{\varphi}+\tilde{\delta})^{\ell^{+}}, \sum_{\eta=1}^{\infty}{ }^{\gamma^{\star \star}+}\left(\tilde{\lambda}_{\eta}+\tilde{\gamma}_{\eta}\right)^{u^{-}}={ }^{\gamma^{\star \star}+}(\tilde{\lambda}+\tilde{\gamma})^{u^{-}} \text {, } \\
& \sum_{\eta=1}^{\infty}{ }^{\gamma^{\star}+}\left(\tilde{\lambda}_{\eta}+\tilde{\gamma}_{\eta}\right)^{u+}={ }^{\gamma^{\star \star}+}(\tilde{\lambda}+\tilde{\gamma})^{u+} \\
& \sum_{\eta=1}^{\infty}{ }^{\gamma^{\star \star}+}\left(\tilde{\varphi}_{\eta}+\tilde{\delta}_{\eta}\right)^{u^{-}}={ }^{\gamma^{\star \star}+}(\tilde{\varphi}+\tilde{\delta})^{u^{-}} \quad \text { and } \quad \sum_{\eta=1}^{\infty}{ }^{\gamma^{\star \star}+}\left(\tilde{\varphi}_{\eta}+\tilde{\delta}_{\eta}\right)^{u^{+}}= \\
& \gamma^{{ }^{\star}+}(\tilde{\varphi}+\tilde{\delta})^{u^{+}} \text {. }
\end{aligned}
$$

Theorem 2.14. For $\left(\left[\tilde{Z}_{\eta}\right]=\left[\tilde{\mu}_{\eta}\right][+] i\left[\tilde{\lambda}_{\eta}\right]\right),\left(\left[\widetilde{\Psi}_{\eta}\right]=\left[\tilde{\gamma}_{\eta}\right][+] i\left[\tilde{\beta}_{\eta}\right]\right),\left(\left[\widetilde{W}_{\eta}\right]\right.$ $=\left[\tilde{\delta}_{\eta}\right][+] i\left[\widetilde{\Delta}_{\eta}\right] \subset\left[\tilde{\mathcal{F}}_{\neg N}^{* *}\right] \quad$ and $\quad\left[\gamma^{\star}, \gamma^{\star \star}\right]+\left[\tilde{\mu}_{\eta}\right] \leq{ }^{\left[\gamma^{\star}, \gamma^{\star \star}\right]+}\left[\tilde{\gamma}_{\eta}\right] \quad, \quad{ }^{\left[\gamma^{\star}, \gamma^{\star \star}\right]+}\left[\tilde{\lambda}_{\eta}\right] \leq$ ${ }^{\left[\gamma^{\star}, \gamma^{\star \star}\right]+}\left[\tilde{\beta}_{\eta}\right], \quad{ }^{\left[\gamma^{\star}, \gamma^{\star \star}\right]+}\left[\tilde{\gamma}_{\eta}\right] \leq{ }^{\left[\gamma^{\star}, \gamma^{\star \star}\right]+}\left[\tilde{\delta}_{\eta}\right], \quad{ }^{\left[\gamma^{\star}, \gamma^{\star \star}\right]+}\left[\tilde{\beta}_{\eta}\right] \leq{ }^{\left[\gamma^{\star}, \gamma^{\star \star}\right]+}\left[\widetilde{\Delta}_{\eta}\right] . \quad$ If GRVBCCCNFNS $\widetilde{\sum_{\eta=1}^{\infty}}\left[\widetilde{Z}_{\eta}\right]$ and $\widetilde{\sum_{\eta=1}^{\infty}}\left[\widetilde{W}_{\eta}\right]$ converge to GRVBCCCNFN $[\tilde{Z}]=$ $[\tilde{\mu}][+] i[\tilde{\lambda}]$. Then $\widehat{\sum_{\eta=1}^{\infty}}\left[\widetilde{\Psi}_{\eta}\right]=[\tilde{Z}]$.

\section{Proof: Obvious.}

\section{Conclusion}

In this paper, we introduced $\tilde{\Gamma}$-converge, $\left[\gamma^{\star}, \gamma^{\star \star}\right]$-level converge, and fuzzy complex sequences and series for new type of fuzzy quantities. We found relationship between $\tilde{\Gamma}$-converge and $\left[\gamma^{\star}, \gamma^{\star \star}\right]$-level converge under some conditions and give connections between GRVBCCCNFNs and CNFNs in terms of $\left[\gamma^{\star}, \gamma^{\star \star}\right]$-level converges. Furthermore, we have checked the basic fuzzy arithmetic operations among GRVBCCCNFNs valued convergences and series. We presented relations of GRVBCCCNFNS with the real and imaginary parts in terms of the fuzzy number series and demonstrated some results between GRVBCCCNFNS with the lower and upper $\gamma$-levels. 


\section{References}

[1] J. J. Buckley and Y. Qu, Fuzzy complex analysis I: Differentiation II: Integration, Fuzzy Sets and Systems 41 (1991) 269-284, 49 (1992) 171-179.

[2] J. J. Buckley, Fuzzy complex numbers, Fuzzy Sets and Systems 33 (1989) 333345 .

[3] Q. P. Cai, The continuity of complex fuzzy function, Adv. in Int. and Soft Computing 62 (2009) 695-704.

[4] S. S. L. Chang, L. A. Zadeh, Fuzzy mappings and control, IEEE Trans. Syst., Man and Cyber., SMC-2, (1972) 30-34.

[5] D. Dubois, and H. Prade, Towards fuzzy differential calculus, Part 1: Integration of fuzzy mappings Part 2: Integration on fuzzy intervals Part 3: Differentiation, FSS 8 (1982) 1-17, 105-116, 225-233.

[6] X. Fu, Q. Shen, fuzzy complex numbers and their application for classifiers performance evaluation, Pattern Recognition 44 (2011) 1403-1417.

[7] Z. Guangquan, Fuzzy limit theory of Fuzzy complex numbers, Fuzzy Sets and Systems 46 (1992) 227-235.

[8] W. Guijun, Y. Shumin, The convergence of fuzzy complex valued series, BUSEFAL, 69 (1997) 156-162.

[9] M. Ha, W. Pedrycz, L. Zheng, The theoretical fundamentals of learning theory based on fuzzy complex random samples, Fuzzy Sets and Systems 160 (2009) 2429-2441.

[10] A. K. Jabbar, M. A. Al-Khafagi, and P. O. Sabir, On Fuzzy Complex Sequences, Journal of University of Duhok, vol 16 (2013).

[11] S. Ma, D. Peng, The fixed point of fuzzy complex number valued mapping AMS 1 (2007) 739-747

[12] D. Qiu, L. Shu, Notes on "On the restudy of fuzzy complex analysis: Part I and Part II", Fuzzy Sets and Systems 159 (2008) 2185-2189. 
[13] J. Qiu, C. Wu, and F. Li, On the restudy of fuzzy complex analysis: Part I. The sequence and series of fuzzy complex numbers and their convergences, Fuzzy Sets and Systems 115 (2000) 445-450.

[14] J. Qiu, C. Wu, and F. Li, On the restudy of fuzzy complex analysis: Part II. The continuity and differentiation of fuzzy complex functions, Fuzzy Sets and Systems 120 (2001) 517-521.

[15] J. Qiu, C. Wu, Some remarks for fuzzy complex analysis, Fuzzy Sets and Systems 106 (1999) 231-238.

[16] P. O. Sabir, A. K. Jabbar, M.A. Al-khafagy, The continuity and differentiation of complex fuzzy functions for new fuzzy quantities, Asian Transactions on Science and Technology, Issue 4 (2) (2012), 1-11.

[17] C. Song, S. Ma, An information algorithm based on segeno fuzzy complex valued integral, Journal of computational Systems 6 (2011) 2166-2171.

[18] G. Wang, X. Li, Generalized Lebesgue integrals of fuzzy complex valued functions, Fuzzy Sets and Systems 127 (2002) 363-370.

[19] E. A. Youness, I. M. Mekawy, A study on fuzzy complex linear programming problems, Int. J. Contemp. Math. Sciences 7 (2012) 897-908.

[20] L. A. Zadeh, Fuzzy sets, Inform. \& Control 8 (1965) 338-353.

[21] L. A. Zadeh, The Concept of a Linguistic Variable and its Application to Approximate Reasoning. Memorandum ERL-M 411, Berkeley, Ca., (1973).

[22] L. A. Zadeh, The concept of a linguistic variable and its application to approximate reasoning (I), (II), and (III), Inf. Sci. 8 (1975) 199-249, 301-357, 9 (1975) 43-80.

[23] Z. Zhang, J. Tian, Structure risk minimization principle based on complex fuzzy random samples, J. of Inf. and Computing Science 5 (2010) 19-40.

[24] L. F. Zheng, M. H. Ha, Further discussions on rectangular fuzzy complex numbers, doi: 10.1109/ICMLC.2009.521 2339. 